\title{
Toxicology and Stem Cells: New Frontiers
}

\author{
Francesca Caloni ${ }^{1}$, Maura Ferrari ${ }^{2}$, and Isabella De Angelis ${ }^{3}$ \\ ${ }^{1}$ Università degli Studi di Milano, Department of Health, Animal Science and Food Safety, Milan, Italy; ${ }^{2}$ Istituto Zooprofilattico \\ Sperimentale della Lombardia e dell'Emilia Romagna "Bruno Ubertini” (IZSLER), Brescia, Italy; ${ }^{3}$ Istituto Superiore di Sanità, \\ Department of Environment and Primary Prevention, Rome, Italy
}

http://dx.doi.org/10.14573/altex.1312021

The annual conference of the Italian Platform on Alternative Methods (IPAM) in collaboration with the Reference Center for Alternative Methods, Care and Welfare of Animals, held at the Brescia Istituto Zooprofilattico Sperimentale della Lombardia e dell'Emilia Romagna "Bruno Ubertini" (ISZLER) on November 14, 2013 was focused on stem cells as a promising tool for toxicology screening. The conference was opened by Dr S. Cinotti (ISZLER) and chaired by Isabella De Angelis, IPAM president, and Francesca Caloni, University of Milan.

Kate Harris, GlaxoSmithKline, gave a presentation on the Comparison of electrophysiological data from human induced pluripotent stem cell derived cardiomyocytes (hiPSC-CMs) to current pre-clinical cardiac safety assays. Cardiotoxicity, notably QT prolongation and torsadogenic risk, is a leading cause of drug attrition during development. Human induced pluripotent stem cell derived cardiomyocytes (hiPSC-CMs) with multi-electrode array (MEA) technology could be used for predictive electrophysiological cardiac safety screening. hiPSC-CMs studied on MEAs were pharmacologically validated using a set of 10 reference compounds and results were compared with other preclinical cardiac safety assays used to assess cardiovascular electrophysiological risk (patch clamping, rabbit ventricular wedge assay, rodent and non-rodent in vivo models). The results showed that hiPSC-CMs demonstrate relevant pharmacological responses and show good correlation to other preclinical assays. Based on this data, hiPSC-CMs could provide an excellent alternative in vitro model to screen for drug induced cardiovascular electrophysiological risk and therefore reduce the number of animals currently used in in vitro studies.

Dr Giovanna Lazzari, Avantea, described the state of the art and perspective of the use of embryonic stem cells as an alternative model in toxicology. The presentation started with a description of the Embryonic Stem Cell Test (EST) for in vitro developmental toxicity and the use of molecular endpoints. The second part of the speech was focused on the EC-funded FP7 project, Embryonic Stem cell based Novel Alternative Testing Strategies (ESNATS), which covers aspects of reproductive toxicity, neurotoxicity, metabolism, and toxicokinetics, following the 3Rs principles, in particular for testing chemical substances and drugs.

Giuliana Pellicci, Istituto Europeo di Oncologia (IEO), described her work on neural stem cells and their possible application in in vitro toxicity studies. The role of the cell surface marker CD133, a cholesterol-interacting membrane protein concentrated in plasma membrane protrusions in cancer stem cells derived from human glioblastoma, and the functional role of the CLIC1 ion channel, localized to the cytosol in glioblastoma derived stem/progenitor cells, were discussed.

Silvia Dotti, IZSLER, presented a summary of the main activities performed at her institute in the field of veterinary regenerative med- icine. In particular, cell therapy based on mesenchymal stem cells (MSCs) was described. These cells are of high interest in human and veterinary medicine, due to their ability to repair damaged tissues and their immunomodulatory properties. The ability of MSCs to repair tendon lesions in the equine species was explained; MSCs demonstrated the ability to grow and to differentiate into three lineages, attesting their pluripotency. They did not cause any immunologic response and none of the treated animals showed adverse effects after MSCs injection. Ecographic examinations showed good regeneration of the tendon. Due to growing interest in the use of MSCs in regenerative therapy, a research project to study in vitro and in vivo biological characteristics of these cells was launched by IZSLER in order to maintain MSCs isolated from bone marrow and adipose tissue and to furnish information about operative protocols. Some innovative approaches on corneal transplants using isolated, amplified ocular stem cells also were described.

The relevance of infrastructure for biomedical research was extensively presented by Francesca Capone, ISS. Translational research is considered by the scientific community, governments, and the public to be a high priority area and many efforts have been focused on the need to promote, at national and international level, specific initiatives to allow more efficient transfer of scientific discoveries into clinical applications. In 2006, the European Strategy Forum on Research Infrastructures (ESFRI) published its first roadmap for the creation of research infrastructure, in which it highlighted the strong need for infrastructure in the field of biomedical sciences. Subsequently, the European Union funded, under FP7, several projects for the establishment of infrastructure to support translational medicine, including European Advanced Translational Research Infrastructure (EATRIS), European Clinical Research Infrastructures Network (Ecrin), and Biobanking and Biomolecular Resources Research Infrastructure (BBMRI). The Italian network of EATRIS, IATRIS, hosted at ISS (http://www.iss.it/iatr/), is particularly active in assistance and technical advice related to regulatory and ethical aspects concerning biomedical advanced technology and the realization of training courses for specialized professional profiles.

The conference concluded with the award "Farmindustria 2013", which is awarded annually to young researchers that are main authors of a scientific paper in the field of alternative methods for research and development of drug products. This year the prize was given to Dr Nadia Ucciferri from Pisa National Research Council for her $\mathrm{PhD}$ thesis "Functional and morphological study of cells in connected culture in response to interactions associated with nanoparticles" in which the innovative development of an advanced in vitro model using a microfluidic-based technology to connect tissue models was described. This model significantly improves the use of dynamic conditions in in vitro toxicity testing. 